\title{
PRIMEIRA OCORRÊNCIA DE GOMPHOTHERIIDAE NO MUNICÍPIO DE IRAUÇUBA, CEARÁ, BRASIL
}

\author{
GINA CARDOSO DE OLIVEIRA \\ Centro de Tecnologia e Geociências, Departamento de Geociências, UFPE, Rua Acadêmico Hélio Ramos, s/n, 50740-530, \\ Recife, PE, Brasil.gina.caroly@hotmail.com \\ MARIA SOMÁLIA SALES VIANA \\ Laboratório de Paleontologia/UVA, Av. Dom José, 878, 62010-290, Sobral, CE, Brasil.somalia_viana@hotmail.com \\ EDISON VICENTE OLIVEIRA \\ Centro de Tecnologia e Geociências, Departamento de Geociências, UFPE, Rua Acadêmico Hélio Ramos, s/n, 50740-530, \\ Recife, PE, Brasil.edison.vicente@ufpe.br
}

\begin{abstract}
FIRST OCCURRENCE OF GOMPHOTHERIIDAE IN IRAUÇUBA MUNICIPALITY, CEARÁ, BRAZIL. Occurrences of gomphotheres in northeastern Brazil there are in tank deposits, caves and lagoons and many of these records are from the Pleistocene tank deposits, formed by physical and chemical erosion in crystalline rock. These deposits are common in northwestern of State of Ceará and correspond to the most Pleistocene fossil findings. This paper presents the description of two specimens of gomphotheres identified as Gomphotheriidae constituting an unprecedented record for the City of Irauçuba, Ceará, located $150 \mathrm{~km}$ from Fortaleza. In the same place, Xenarthra, Liptoterna, Notoungulata and Perissodactyla were also found.
\end{abstract}

Key words: proboscideans, new record, Irauçuba, Ceará, Brazil.

\section{INTRODUÇÃO}

Atualmente são referidas três espécies de proboscídeos na América do Sul: Amahuacatherium peruvium Campbell, Frailey \& Romero-Pittman, 2000, Cuvieronius hyodon (Fischer, 1814) e Notiomastodon platensis (Ameghino, 1888). A. peruvium tem seu registro no Mioceno do Peru, entretanto a sua validade é questionada por alguns autores (e.g. Woodburne, 2010; Lucas, 2013). A espécie C. hyodon é registrada na Bolívia e Peru, enquanto $N$. platensis pelo continente sul-americano, nas planícies e também em regiões de grandes altitudes (Mothé \& Avilla, 2013). Esses táxons têm várias características compartilhadas, como mandíbula brevirrostrina, um par de incisivos superiores e molares bunodontes, sendo basicamente diferenciados pela torção das defesas superiores em $C$. hyodon, e complexidade dos molares mais acentuada em $N$. platensis.

No Nordeste do Brasil são registrados proboscídeos desde o século XVIII, que foram preservados em depósitos quaternários de tanque, lagoas, e em cavernas (Paula-Couto, 1979; Cartelle, 1994; Fernandes et al., 2013). Brasil (1863) mencionou a ocorrência de mastodonte para o Estado do Ceará, mas não fez referência da localidade. Posteriormente, outros autores citaram ocorrências de mastodontes para o Estado do Ceará nos municípios de Itapipoca, Quixadá, Cascavel e Limoeiro do Norte (Moraes, 1924); Baturité, Mombaça, Jaguaribe, Tauá e Arneiroz (Simpson \& Paula Couto, 1957); Sobral (Nascimento et al., 1981); Fortaleza (Leonardos, 1946; Oliveira, 2010); Senador Pompeu (Silva Santos, 1982; Oliveira, 2010). Até o momento, o material melhor conhecido de proboscídeo do Ceará está em Simpson \& Paula Couto (1957), porém sem figuração dos fósseis e com breve descrição. O presente estudo tem por objetivo registrar a ocorrência de Gomphotheriidae no Município de Irauçuba, região centro-norte do Estado do Ceará.

\section{MATERIAL E MÉTODOS}

O material estudado trata-se de um dentário direito incompleto com $\mathrm{M}_{3}$ desgastado (MDJ-M-Irauçuba-050) e um fêmur direito (MDJ-M-Irauçuba-052), os quais foram coletados por moradores da região, em outubro de 2012 no Distrito de Juá, no Município de Irauçuba, Lagoa das Pedras (41'49,8”S/39 50’20,1'”) (Figura 1). A equipe do Laboratório de Paleontologia da Universidade Estadual Vale do Acaraú/Museu Dom José foi até o local analisar a área de escavação (Figura 2), com aproximadamente $2 \mathrm{~m}^{2}$ por cerca de $4 \mathrm{~m}$ de profundidade até o nível da água. O material estudado estará sob a guarda do MDJ até que seja montado o Museu do Município de Irauçuba, o qual consta do planejamento municipal.

A identificação dos fósseis seguiu Paula Couto (1979), Simpson \& Paula Couto (1957) e Osborn (1936, 1942). As medidas foram tomadas segundo Proaño (1922) e Ferretti (2010) (Figura 3). Foi utilizado para comparação o material de mastodontes pertencente ao acervo do Museu Dom José, Sobral, bem como aqueles descritos por Gadens-Marcon (2008) e Dantas (2004). Para a classificação do grau de desgaste dentário foi utilizada a tabela de Simpson \& Paula- 


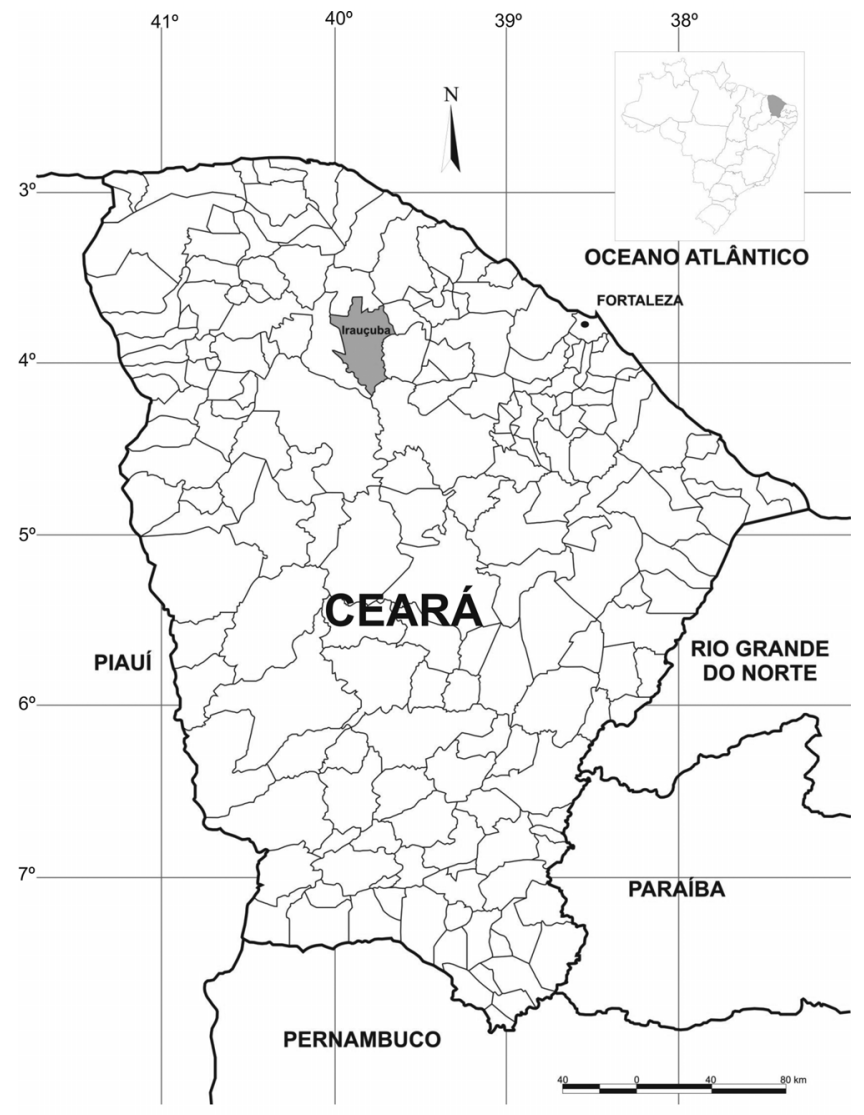

Figura 1. Mapa de localização do Município de Irauçuba (cinza), Estado do Ceará, Brasil.

Figure 1. Location map of the Irauçuba Municipality (gray), State of Ceará, Brazil.

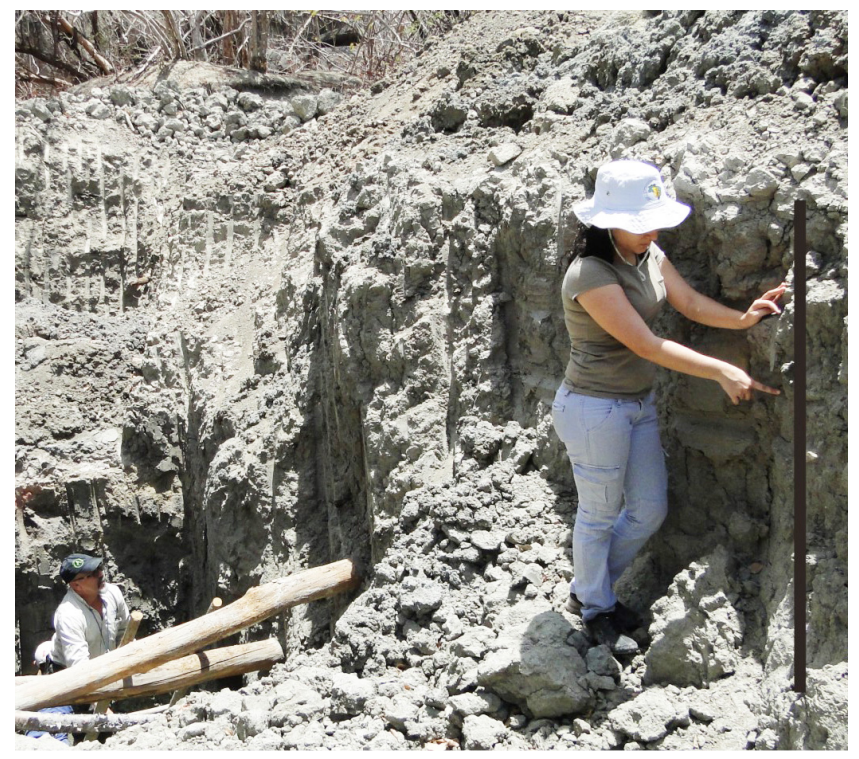

Figura 2. Tanque em Irauçuba. Escavação atual e nível de aproximadamente $1,5 \mathrm{~m}$ contendo alguns fragmentos de fósseis (barra preta).

Figure 2. Tank in Irauçuba. Current excavation and level with approximately $1.5 \mathrm{~m}$ containg some fossil fragments (black bar).
Couto (1957).

Abreviaturas institucionais. IHGS, Instituto Histórico e Geográfico de Sergipe, Aracaju, SE; LABOPALEO/UVA/ MDJ, Laboratório de Paleontologia da Universidade Estadual Vale do Acaraú/Museu Dom José, Sobral, CE, Brasil; MCNPV, Coleção de Paleontologia de Vertebrados do Museu de Ciências Naturais da Fundação Zoobotânica do Rio Grande do Sul, Porto Alegre, RS, Brasil.

\section{SISTEMÁTICA PALEONTOLÓGICA}

Ordem PROBOSCIDEA Illiger, 1811

Família GOMPHOTHERIIDAE Hay, 1922

(Figuras 4-5)

Material. MDJ-M-Irauçuba-050, dentário direito incompleto $\mathrm{M}_{3}$ desgastado; MDJ-M-Irauçuba-052, fêmur direito.

Proveniência geográfica/idade. Irauçuba, Ceará, Brasil; Pleistoceno.

Descrição. O dentário MDJ-M-Irauçuba-050 (Figura 4) apresenta um $\mathrm{M}_{3}$ pentalofodonte em estágio 4 (desgaste severo, padrão parcial ou totalmente destruído) de desgaste e parte anterior levemente fragmentada. Alvéolo do $\mathrm{M}_{1} \mathrm{em}$ fase de fechamento. Sínfise mandibular incompleta e ramo mandibular ausente, perdidos provavelmente durante a coleta. Possui uma altura máxima, ao nível do $\mathrm{M}_{3}$, de $170 \mathrm{~mm}$ e comprimento de $390 \mathrm{~mm}$. Na face lateral externa está inserido o forame mentoniano, logo abaixo do alvéolo, e apresenta $20 \mathrm{~mm}$ de diâmetro. $\mathrm{O}$ corpo mandibular é convexo externamente e internamente; largo na porção medial e estreitando-se à medida que chega à região sinfisiária, da mesma forma que chega à região do ramo ascendente. O fêmur MDJ-M-Irauçuba-052 (Figura 5) está em perfeito estado de preservação, sendo perdidos apenas alguns fragmentos durante a coleta. Apresenta $900 \mathrm{~mm}$ de comprimento, cuja diáfise é achatada ântero-posteriormente, sendo mais larga que espessa. As extremidades apresentam epífises fusionadas sugerindo um indivíduo adulto. A largura da extremidade proximal é de $340 \mathrm{~mm}$ e distal, 220 $\mathrm{mm}$. O trocânter menor é reduzido e abaixo do trocânter maior é observada a fossa trocantérica, evidenciada por sua profundidade significativa. A cabeça do fêmur é mais elevada que o trocânter maior, sendo também, evidentemente, mais larga e separada do corpo pelo colo do fêmur. Na face anterior, nota-se um forame mais proximalmente. Há uma curvatura bem evidente na porção medial, alargando-se na porção distal, onde está situado o epicôndilo medial. Abaixo do trocânter menor, há um sulco de $130 \mathrm{~mm}$ de comprimento por $20 \mathrm{~mm}$ de largura, bem superficial e rugoso. Na porção lateral, também há uma curvatura, mais curta, começando a partir do terceiro trocânter, não bem evidenciado, até o epicôndilo lateral. O epicôndilo lateral é maior próximodistalmente e médio-lateralmente, em relação ao epicôndilo medial. A fossa intercondilar é bem profunda, com vários forames no seu interior.

Comparação. O dentário MDJ-M-Irauçuba-050 apresenta dimensões similares ao do espécime MCN-PV 7089 descrito 
A

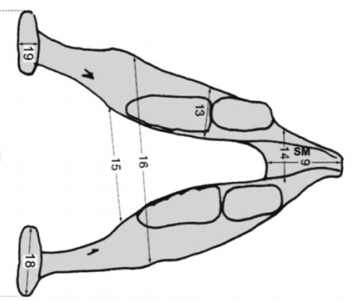

B
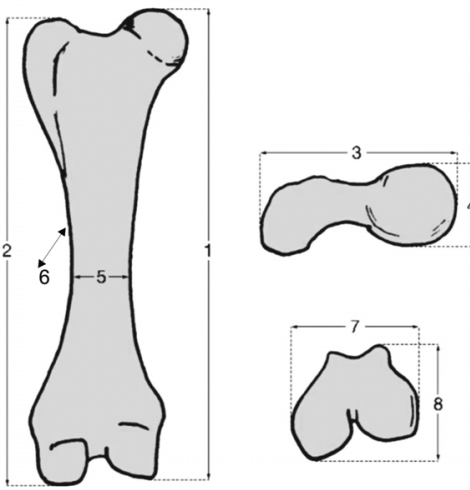

Figura 3. Medidas de mandíbula (A) e fêmur (B) segundo Proaño (1922). Abreviaturas: C, côndilo; SM, sínfise mandibular; PC, processo coronoide; RI, região infradental; RA, ramo ascendente; $\mathbf{C M}$, corpo mandibular; TR, trígono retromolar; $\mathbf{F M}$, forame mentoniano. As medidas se encontram nas Tabelas 1 e 2.

Figure 3. Measures of the mandible (A) and femur (B) according to Proaño (1922). Abbreviations: C, condyle; SM, mandibular symphysis; PC, conoid process; RI, infradental region; RA, ascending ramus; CM, mandibular body; TR, retro-molar trigon; FM, mental forame. The measurements are in the Tables 1 and 2.

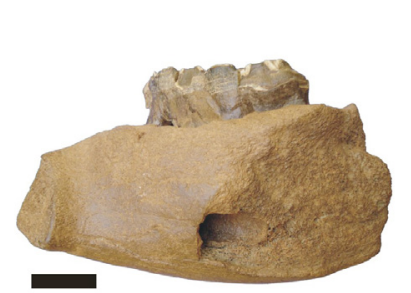

A

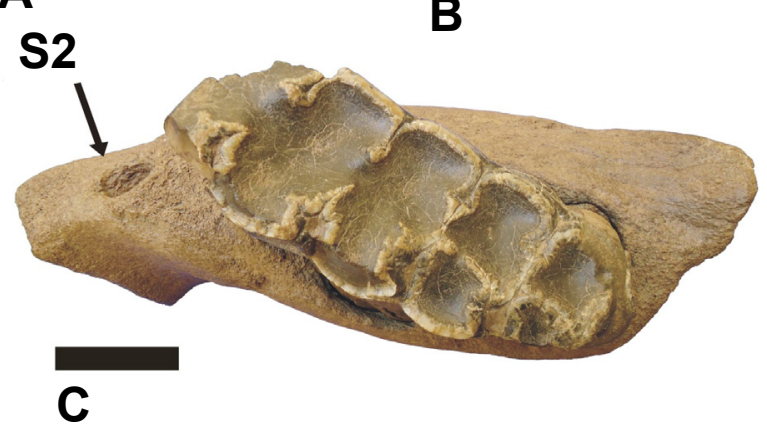

Figura 4. Gomphotheriidae (050-IR). Dentário direito $\operatorname{com} \mathrm{M}_{3}$, vistas medial (A); lateral (B); oclusal (C). Abreviações: S1, forame mentoniano; S2, alvéolo sendo remodelado. Escalas $=100 \mathrm{~mm}$.

Figure 4. Gomphotheriidae (050-IR). Right dentary with $\mathrm{M}_{3}$, medial (A); lateral (B); occlusal (C) views. Abbreviations: S1, mental forame; S2, alveolus being remodeled. Scale bars $=100 \mathrm{~mm}$.

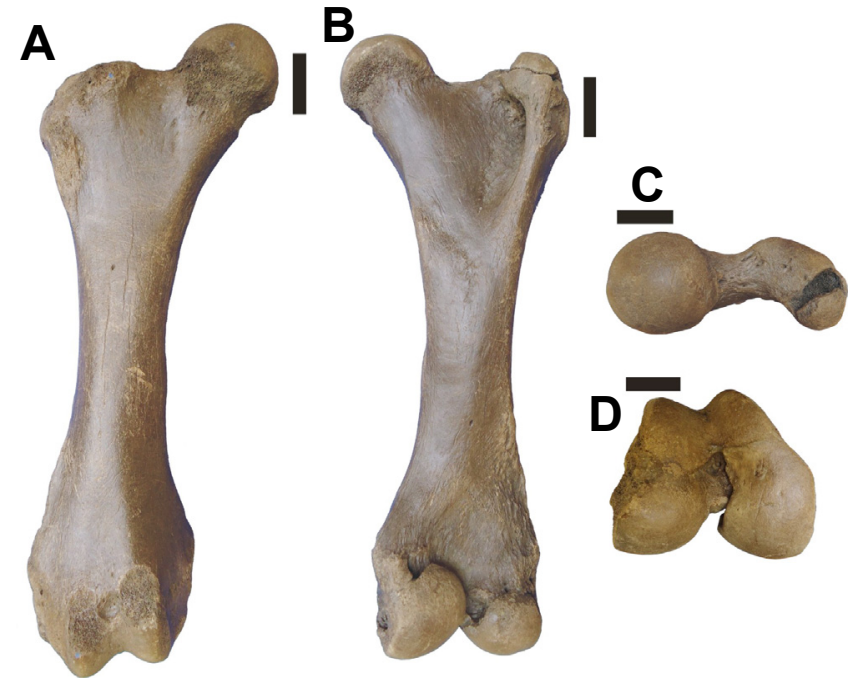

Figura 5. Gomphotheriidae (052-IR). Fêmur direito, vista anterior (A); posterior (B); proximal (C); distal (D). Escalas $=100 \mathrm{~mm}$.

Figure 5. Gomphotheriidae (052-IR). Right femur, anterior (A); posterior (B); proximal (C); distal (D) views. Scale bars $=100 \mathrm{~mm}$.

Tabela 1. Medidas ( $\mathrm{mm}$ ) do dentário. Veja Figura 3.

Table 1. Measurements $(\mathrm{mm})$ of the dentary. See Figure 3.

\begin{tabular}{lc}
\hline \multicolumn{1}{c}{ Medidas } & Espécime 050-IR \\
\hline $\begin{array}{l}\text { 4. Comprimento: origem infradental anterior do } \\
\text { ramo ascendente }\end{array}$ & 390 \\
$\begin{array}{l}\text { 5. Comprimento infradental oral da borda do } \mathrm{M}_{3} \\
\begin{array}{l}\text { 7. Comprimento: borda oral do } \mathrm{M}_{3}-\text { origem } \\
\text { anterior do ramo ascendente }\end{array}\end{array}$ & 270 \\
$\begin{array}{l}\text { 12. Altura do corpo do dentário no ponto médio } \\
\text { da linha do molar }\end{array}$ & 170 \\
$\begin{array}{l}\text { 13. Espessura maior do corpo do dentário do } \\
\text { ponto médio da linha do molar }\end{array}$ & 180 \\
\hline
\end{tabular}

Tabela 2. Medidas (mm) do fêmur. Veja Figura 3.

Table 2. Measurements ( $\mathrm{mm}$ ) of the femur. See Figure 3.

\begin{tabular}{lcc}
\hline \multicolumn{1}{c}{ Medidas } & $\begin{array}{c}\text { Espécime } \\
052-I R\end{array}$ & $\begin{array}{c}\text { Espécime } \\
\text { IHGS } \\
\text { 2004/00143 }\end{array}$ \\
\hline 1. Maior comprimento da cabeça & 900 & - \\
2. Comprimento lateral & 870 & - \\
3. Maior amplitude da extremidade proximal & 340 & - \\
4. Maior profundidade da cabeça & 145 & - \\
5. Menor amplitude da diáfise & 140 & - \\
6. Espessura da diáfise & 90 & - \\
7. Maior amplitude da extremidade distal & 220 & 180 \\
8. Profundidade da extremidade distal & 250 & 200 \\
\hline
\end{tabular}


por Gadens-Marcon (2008) para o Rio Grande do Sul. Já quando comparados com MDJ-M 122 e MDJ-M 026 (Oliveira, 2011), o espécime de Irauçuba apresenta medidas superiores e é mais robusto (Tabela 1), porém isto pode tratarse de uma variação intraespecífica. $\mathrm{O}$ único molar encontrado no dentário é um $\mathrm{M}_{3}$ bastante desgastado, o que indica tratarse de um indivíduo de idade avançada. O fêmur MDJ-MIrauçuba-052 têm medidas semelhantes aos dos espécimes do Rio Grande do Sul, sendo às vezes de tamanho superior (Tabela 2). As medidas 7 e 8, da extremidade distal, são superiores ao do espécime IHGS 2004/00143 de Aquidaban, Estado de Sergipe, descrito por Dantas (2004).

\section{DISCUSSÃO}

A morfologia, assim como as variações de dimensões encontradas nos espécimes estudados estão dentro do padrão esperado para Gomphotheriidae e, portanto, registra-se pela primeira vez a ocorrência desta família para o Município de Irauçuba, Estado do Ceará. Entretanto, a maioria dos caracteres utilizada para a classificação genérica e específica dos gonfoterídeos por alguns autores (e.g. Alberdi et al., 2002; Ferreti, 2010; Mothé et al, 2011; Lucas, 2013) é encontrada no crânio e dentição com padrão de desgaste bem evidente, o que não é o caso dos espécimes aqui descritos. Com relação ao pós-crânio, não há estudos que atribuam características pós-cranianas para a identificação específica de proboscídeos sul-americanos. Portanto, mesmo com o bom estado de conservação do material fóssil, este Gomphotheriidae não foi atribuído à Notiomastodon platensis, pois apesar de ter sido encontrado em área onde esta é a única espécie registrada, os espécimes não preservam as suas características diagnósticas.

\section{AGRADECIMENTOS}

Os autores agradecem a F. Irani, pela doação do material ao MDJ; à CAPES, pelo auxílio financeiro através de bolsa de mestrado concedida à primeira autora.

\section{REFERÊNCIAS}

Alberdi, M.T. \& Prado, J.L. 1995. Los Mastodontes de América del Sur. In: M.T. Alberdi; G. Leone \& E.P. Tonni (eds.) Evolución biológica y climática de la Región Pampeana durante los últimos 5 millones de años. Un ensayo de correlación con el Mediterráneo occidental. Madrid, Museo Nacional de Ciencias Naturales, CSIC, p. 277-292 (Monografías 12).

Alberdi, M.T.; Prado, J.L. \& Cartelle, C. 2002. El registro de Stegomastodon (Mammalia, Gomphotheriidae) en el Pleistoceno superior de Brasil. Revista Española de Paleontología, 17:217-235.

Brasil, T.P.S. 1863. Ensaio estatístico da província do Ceará. (Tomo I). Fortaleza, Tipografia B. de Matos, 839 p.

Dantas, M.A.T. 2004. Os fósseis da megafauna pleistocênica do Instituto Histórico e Geográfico de Sergipe. Canindé, 4:383-393.
Ferretti, M.P. 2010. Anatomy of Haplomastodon chimborazi (Mammalia, Proboscidea) from the late Pleistocene of Ecuador and its bearing on the phylogeny and systematics of South American gomphotheres. Geodiversitas, 32:663-721. doi:10.5252/g2010n4a3

Gadens-Marcon, G.T. 2008. Contribuição ao estudo dos Proboscidea (Mammalia, Gomphotheriidae) do Quaternário do Estado do Rio Grande do Sul, Brasil. Revista Geociências - UnG, 7:93-109.

Hoffstetter, R. 1952. Les mammiféres plesitocènes de la République del Equateur. Memoires de La Société Géologique de France, 66:1-391.

Lucas, S.G. 2013. The palaeobiogeography of South American gomphotheres. Journal of Palaeogeography, 2:19-40. doi:10.3724/SP.J.1261.2013.00015

Lucas, S.G. \& Alvarado, G.E. 2010. Fossil Proboscidea of the upper Cenozoic of central America: taxonomy, evolutionary and paleobiogeographic significance. Revista Geologica de America Central, 42:9-42.

Moraes, L.J. 1924. Serras e montanhas do Nordeste. Rio de Janeiro, Inspetoria Federal de Obras Contra as Secas, p. 51-58 (Coleção Mossoroense 35).

Mothé, D. \& Avilla, L.S. 2013. Derrubando "mitos" sobre os Gonfoterídeos Sulamericanos (Proboscidea, Gomphotheriidae). In: CONGRESSO BRASILEIRO DE PALEONTOLOGIA, 23 , 2013. Boletim de Resumos, Gramado, p. 250-251.

Mothé, D.; Avilla, L.S.; Cozzuol, M. \& Winck. G.R. 2011. Taxonomic revision of the Quaternary gomphotheres (Mammalia: Proboscidea: Gomphotheriidae) from the South American lowlands. Quaternary International, 276-277:2-7. doi:10.1016/j. quaint.2011.05.018

Nascimento, D.A.; Gava, A.; Pires, J. \& Teixeira, W. 1981. Geologia da folha SA. 24 - Fortaleza. In: Projeto Radambrasil, DNPM, p. 23-212.

Oliveira, P.V. 2010. Mamíferos do neopleistoceno-Holoceno do Parque Nacional de Ubajara, Ceará. Programa de Pósgraduação em Geociências, Universidade Federal do Rio Grande do Sul, Dissertação de Mestrado, 166 p.

Osborn H.F. 1936. Proboscidea. New York, American Museum of Natural History, v. 1, 802 p.

Osborn, H.F. 1942. Proboscidea. New York, American Museum of Natural History, v. 2, 960 p.

Paula-Couto, C. 1970. Evolução de comunidades, modificações faunísticas e integrações biocenóticas dos vertebrados cenozóicos do Brasil. In: CONGRESSO LATINO DE ZOOLOGIA, 4, 1992. Actas, Caracas, p. 907-930.

Paula-Couto, C. 1979. Tratado de Paleomastozoologia. Rio de Janeiro, Academia Brasileira de Ciências, 590 p.

Shoshani, J. \& Thassy, P. 2005. Advances in proboscidean taxonomy and classification, anatomy and physiology, and ecology and behavior. Quaternary International, 126-128:5-20. doi:10.1016/j.quaint.2004.04.011

Simpson, G.G. \& Paula-Couto, C. 1957. The Mastodonts of Brazil. Bulletin of the American Museum of Natural History, 112:125-190.

Woodburne, M.O. 2010. The Great American Biotic Interchange: dispersals, tectonics, climate, sea level and holding pens. Journal of Mammalian Evolution, 17:245-264. doi:10.1007/ s10914-010-9144-8

Received in January, 2014; accepted in May, 2015. 